\title{
Resonant two-beam interferometric sensor independent of intracavity losses
}

\author{
Marco Romanelli \\ marco.romanelli@univ-rennes1.fr \\ Marc Vallet \\ marc.vallet@univ-rennesı.fr
}

\author{
Institut de Physique de Rennes, UMR CNRS 6251, Université de Rennes 1, Campus de Beaulieu, 35042 \\ Rennes, France \\ Institut de Physique de Rennes, UMR CNRS 6251, Université de Rennes 1, Campus de Beaulieu, 35042 \\ Rennes, France
}

We study a resonant interferometric sensor with two cavity eigenstates. The finesse associated to one eigenstate is significantly lower than the empty cavity value, because of the presence of a lossy intracavity sample. We show theoretically that the sensitivity of the interferometer only depends on the empty cavity finesse when the low-finesse eigenfrequency is locked to resonance. This is experimentally demonstrated and a resolution of $60 \mathrm{pm}$ is reported. Our method can be applied to any resonant two-beam interferometer. [D0I: 10.2971/jeos.2009.09023]

Keywords: interferometry, Fabry-Perot, locking, optical phase measurement

\section{INTRODUCTION}

Two-beam interference by division of amplitude is ubiquitously applied for measuring small optical path difference $(O P D)$ and many interferometers have been developed with sensitivity routinely reaching the nanometer range. The experimental setup usually presents two arms: one probes the effect to be measured (measurement arm) and the other one is used as the reference (reference arm) [1]. In order to enhance the sensitivity of two-beam interferometers, one can combine them with resonant Fabry-Perot (FP) cavities. For example, in gravitational waves interferometric detectors, two cavities of high finesse $F$ are placed inside the two arms of a Michelson interferometer $[2,3]$. This leads to an increase of the order of $F$ for the sensitivity. Another possible configuration is a "resonant two-beam interferometer", i.e., a two-beam interferometer placed inside a resonant cavity [4]. The reference and measurement arms of the two-beam interferometer are then associated with the two spatially separated eigenstates of the cavity [5]. Compared to the OPD associated with the two-beam interferometer, the multiple round-trips in the cavity yield an effective $O P D$ multiplied by $F$ [6]. In order to keep a high sensitivity, that is, a high finesse, the losses inside the cavity have to be reduced as much as possible [7]. This task is straightforward to perform when the samples under study are in gas phase, but for liquid or solid samples, residual absorption or even Fresnel reflections at the interfaces can have a deleterious impact on the finesse and must be eliminated with a careful design of the experimental setup [8,9]. In the context of resonant two-beam interferometers, the situation is somewhat different since the sample is inserted in the measurement arm only. The aim of this paper is to show that in such a case the performance of the apparatus is independent of the intracavity losses, when the measurement arm eigenfrequency is locked to the input optical frequency.

Therefore the use of a resonant two-beam interferometer in- creases the sensitivity with respect to conventional cavityenhanced measurements.

This article is organized as follows. In Section 2, we theoretically address the problem of a generic two-beam resonant interferometer with eigenstates of different finesses. We also analyze the effect of the servo-loop on the resolution associated with the measurement, in the case of a modulationdemodulation scheme. In Section 3, the theoretical predictions are experimentally checked and discussed.

\section{THEORETICAL ANALYSIS}

Without loss of generality, we illustrate the case of two-finesse resonant interferometers with a Jamin-Fabry-Perot interferometer. This resonant two-beam interferometer has been recently demonstrated [4] and successfully employed to study the gravity-driven dynamics of soap film thickness [10]. It consists in a FP cavity containing a polarization-split Jamin interferometer, as shown in Figure 1. Two intracavity polarization beam displacers $(P B D s)$ produce spatially separated eigenstates with associated measurement and reference crosspolarized eigenfields, $\vec{E}_{m}$ and $\vec{E}_{r}$ respectively. Only $\vec{E}_{m}$ probes the intracavity sample $S$. The whole apparatus is sandwiched between two polarizers $P_{1}$ and $P_{2}$. The output field $\vec{E}_{\text {out }}$ impinging on the detector results from the coherent sum of the transmitted measurement and reference fields [11]. Its amplitude writes $[1,11]$ :

$$
\begin{aligned}
E_{\text {out }}=E_{\text {in }}[\cos \theta & T \sqrt{T_{s}} e^{i \varphi_{m}} \frac{1}{1-R T_{S} e^{i 2 \varphi_{m}}} \\
& \left.-\sin \theta T e^{i \varphi_{r}} \frac{1}{1-R e^{i 2 \varphi_{r}}}\right] .
\end{aligned}
$$

$E_{\text {in }}$ is the input field amplitude. $R$ and $T$ are respectively the reflectivity and transmission in intensity of the two identical 


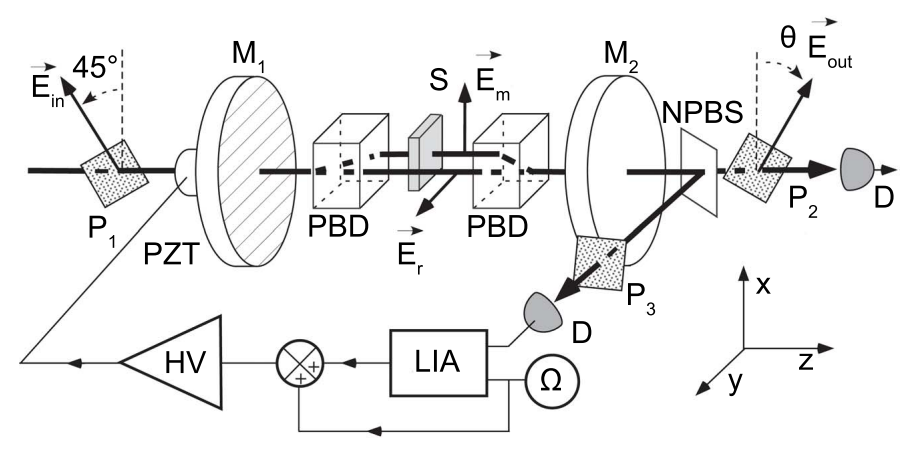

FIG. 1 Scheme of the two-finesse resonant interferometer under study: $P_{i}$, polarizers; $M_{1}, M_{2}$, cavity mirrors; $P B D$, polarization beam displacer; $S$, sample; NPBS, non-polarizing beam splitter; $D$, detector; $P Z T$, piezoelectric transducer; $H V$, highvoltage amplifier; $L I A$, lock-in amplifier operating at reference frequency $\Omega / 2 \pi$.

cavity mirrors $M_{1}$ and $M_{2} . T_{S}$ is the transmission in intensity of $S . \varphi_{m}$ and $\varphi_{r}$ are the phase shifts between $M_{1}$ and $M_{2}$ for the measurement arm and for the reference arm respectively. Notice that residual absorption $A$ associated to the $P D B$ s can be taken into account by putting $(1-A) R$ and $\sqrt{1-A} T$ instead of $R$ and $T$ in Eq. (1). $\theta$ is the angle of the output polarizer axis with respect to the measurement polarization direction. We set $\theta=\tan ^{-1}\left(\frac{(1-R) \sqrt{T_{s}}}{1-R T_{s}}\right)$ in order to equalize the output intensities maxima to optimize the interference visibility. Eq. (1) yields:

$I_{\text {out }}=K^{2} I_{\text {in }}\left|e^{i \varphi_{m}}\left(\frac{1-R T_{S}}{1-R T_{s} e^{i 2 \varphi_{m}}}\right)-e^{i \varphi_{r}}\left(\frac{1-R}{1-R e^{i 2 \varphi_{r}}}\right)\right|^{2}$,

where $K=\frac{1}{\sqrt{2}} \sin \theta$. Eq. (2) expresses the fact that the output intensity results from the interference of the two cavity eigenstates [5]. The two terms in brackets correspond to the two associated Airy functions, with finesses $F_{m}=\frac{\pi \sqrt{R T_{s}}}{1-R T_{s}}$ and $F_{r}=\frac{\pi \sqrt{R}}{1-R}$ respectively. To measure $I_{\text {out }}$, the cavity resonant frequency has to be controlled. This is performed in our setup by servo-locking one eigenfrequency of either the reference arm or the measurement arm to the optical input frequency. Formally, these two possibilities correspond to setting $\varphi_{r}$ or $\varphi_{m}$ equal to zero in Eq. (2).

Figure 2(a) displays the theoretical transmitted intensity as a function of the optical path difference OPD $=\left(\varphi_{m}-\varphi_{r}\right) \frac{\lambda}{\pi}$, where $\lambda$ refers to the optical wavelength. $R, T$ and $T_{S}$ were set to $0.9,0.1$ and 0.7 respectively. This gives $F_{r}=30$ and $F_{m}=6.7$. When OPD is equal to zero, the transmitted signal is zero as a result of destructive interference between the measurement and reference beams. As OPD increases, one obtains a different response, depending on which beam has been chosen for locking the cavity to resonance. The best sensitivity $\Sigma$ corresponds to the maximum value of $\left|\frac{d I}{d O P D}\right|$. Figure 2(b) shows that $\Sigma$ is better when the lower finesse arm is locked to resonance. A straightforward calculation using Eq. (2) yields $\Sigma_{m} / \Sigma_{r}=F_{r} / F_{m}$, where $\Sigma_{i}$ is the best sensitivity obtained when $\varphi_{i}$ is locked to zero. Actually, when one arm eigenfrequency is locked to resonance, the associated Airy function is identically equal to 1 in Eq. (2) and $I_{o u t}$ is independent of its finesse. The other arm acts as a "meter". Therefore, when the broad measurement peak is locked to resonance, the nar-

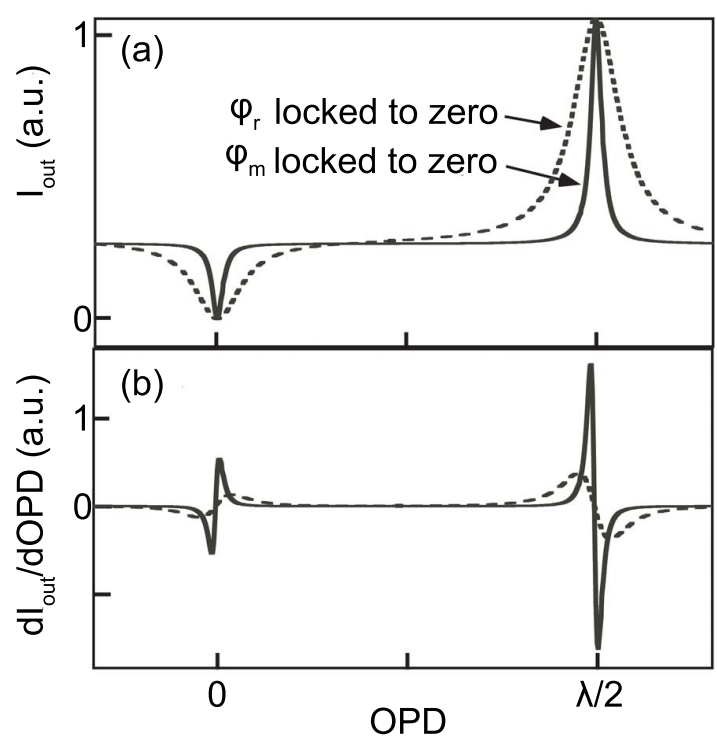

FIG. 2 (a) Output intensity $I_{\text {out }}$ as a function of the optical path difference $O P D$, calculated from Eq. (2), with $R=0.9, T=0.1, T s=0.7$, when the cavity is resonant either for the measurement arm (dashed line), or for the reference arm (solid line). (b) Derivative of $I_{\text {out }}$ as a function of $O P D$.

row reference peak is shifted as $O P D$ changes. The benefit is that the sensitivity is not affected by the poor finesse of the measurement arm, mainly determined by the presence of the sample. Only the reference arm finesse determines the quality of the measurement.

We now discuss the influence of the finesse of the peak locked to resonance on the error signal of the servo-loop. In our setup, a modulation-demodulation scheme is implemented (see Figure 1). The cavity length $L$ is modulated at frequency $\Omega / 2 \pi$ around resonance via a piezoelectric transducer PZT supporting the mirror $M_{1}$. The output beam is partly reflected by a non polarizing beam splitter of reflectivity $R_{N P B S}$ followed by an orientable polarizer $P_{3}$. By suitably orientating $P_{3}$, either the measurement or the reference polarization intensity $I_{f}$ is detected, then demodulated by coherent detection, and finally amplified and fed back to PZT. At resonance, the intensity $I_{f}$ is equal to $I_{0}=\frac{F^{2} T^{2} R_{N P B S}}{\pi^{2} R} I_{i n}$, where $F$ refers to either $F_{m}$ or $F_{r}$. If $\varphi$ is the phase difference from exact resonance, and assuming $\varphi \ll \frac{\pi}{2 F}, I_{f}$ is then given by [1]:

$$
I_{f}=\frac{I_{0}}{1+\left(\frac{2 F}{\pi}\right)^{2} \sin ^{2} \varphi} \simeq I_{0}\left[1-\left(\frac{2 F}{\pi}\right)^{2} \varphi^{2}\right] .
$$

We write $\varphi(t)$ as $\varphi(t)=\varphi_{\varepsilon}(t)+\varphi_{f}(t)+\varphi_{m} \cos (\Omega t) \cdot \varphi_{\varepsilon}(t)$ represents erratic phase fluctuations, $\varphi_{f}(t)$ is the correction proportional to the feedback voltage, and $\varphi_{m} \cos (\Omega t)$ is the modulation at frequency $\Omega / 2 \pi$. The signal component at frequency $\Omega / 2 \pi$ is fed back to the cavity mirror via an amplifier HVA. This latter is supposed to consist of a proportional stage with gain $-G$. The phase correction is then equal to:

$$
\varphi_{f}=-G\left[I_{0}\left(\frac{2 F}{\pi}\right)^{2}\left(\varphi_{\varepsilon}+\varphi_{f}\right) \varphi_{m}+\xi \Omega \frac{\Delta \Omega}{2 \pi}\right],
$$

where $\xi_{\Omega} \frac{\Delta \Omega}{2 \pi}$ is a noise term proportional to the detection bandwidth $\frac{\Delta \Omega}{2 \pi}$. This contribution is homogeneous to an optical intensity and includes the laser intensity noise as well 
as detection noise integrated over the detection bandwidth around the modulation frequency. From Eq. (4) and in the limit $G I_{0}\left(\frac{2 F}{\pi}\right)^{2} \varphi_{m} \gg 1$, we obtain:

$$
\varphi_{f} \simeq-\varphi_{\varepsilon}-\frac{1}{\left(\frac{2 F}{\pi}\right)^{2} \varphi_{m}} \frac{\xi_{\Omega} \Delta \Omega}{2 \pi I_{0}} .
$$

Eq. (5) shows that the phase fluctuations $\varphi_{\varepsilon}$ are perfectly compensated by the feedback loop over the whole feedback bandwidth. Furthermore, the response to phase fluctuations is independent of $F$. Conversely, the response to the equivalent relative intensity noise $\frac{\tilde{s} \Omega}{2 \pi I_{0}}$ depends on $F^{2}$. In the case of our two-finesse Fabry-Perot interferometer, when locking the measurement peak to resonance, one can get the same loop performance with respect to locking the reference peak by keeping constant the product $F^{2} \varphi_{m}$, i.e. by increasing the cavity length modulation. Therefore, servo-locking the low finesse peak to resonance does not degrade the quality of the measurement, as is experimentally shown in the following section.

\section{EXPERIMENTAL RESULTS AND DISCUSSION}

Our setup consists of a $L=20 \mathrm{~cm}$ FP confocal cavity containing two $20 \mathrm{~mm}$ long anti-reflection coated $Y \mathrm{VO}_{4}$ birefringent crystals. The finesse is equal to 30 . The cavity is injected by a frequency-stabilized single-mode laser (Innolight, $\lambda=1064 \mathrm{~nm}$ ). The incident beam polarization is set at $45^{\circ}$ with respect to the crystal neutral axes. The sample consists of a BK7 glass plate of variable thickness, mounted on a micrometric translation stage. The recombined beams are analyzed with polarizer $P_{2}$ followed by a photodiode $D$. The experimental procedure is as follows. First, the cavity is locked to resonance, either for the measurement or the reference arm. Locking is achieved with the modulation-demodulation technique detailed in Section 2. The modulation frequency $\Omega / 2 \pi$ is equal to $93 \mathrm{~Hz}$, and the lock-in integration time is equal to $0.3 \mathrm{~s}$, leading to $\Delta \Omega / 2 \pi=0.5 \mathrm{~Hz}$. Second, one $P B D$ is slightly tilted to set the relative phase shift to zero, thus acting as an adjustable optical bias [11]. $P_{2}$ is then rotated to get the extinction of the transmitted signal. The interferometer is thus in the so-called "dark-field" configuration. Then, $I_{\text {out }}$ is recorded as the $O P D$ is changed by vertically translating the intracavity sample. The experimental results are shown in Figure 3. Each point corresponds to an average over 30 consecutive acquisitions. The product $F^{2} \varphi_{m}$ was kept constant in both configurations. $I_{o u t}$ is plotted as a function of $O P D=(n-1) \Delta e$, where $n$ is the index of refraction and $\Delta e$ is the thickness variation of the glass plate. Figure 3 clearly shows that the interferometer transmission depends on whether one locks the cavity on the measurement or on the reference peak. The best sensitivity is given by the maximal slope of the curve. Therefore one finds that locking the broad measurement peak to resonance leads to a better sensitivity, in agreement with the theoretical predictions of Section 2. The ratio $\Sigma_{m} / \Sigma_{r}$ is equal to 2.3, corresponding to the ratio $F_{r} / F_{m}$. It is in agreement with the measured values $F_{m}=13$ and $T_{s}=0.86$. The noises in the two configurations are shown in the inset of Figure 3. We find that

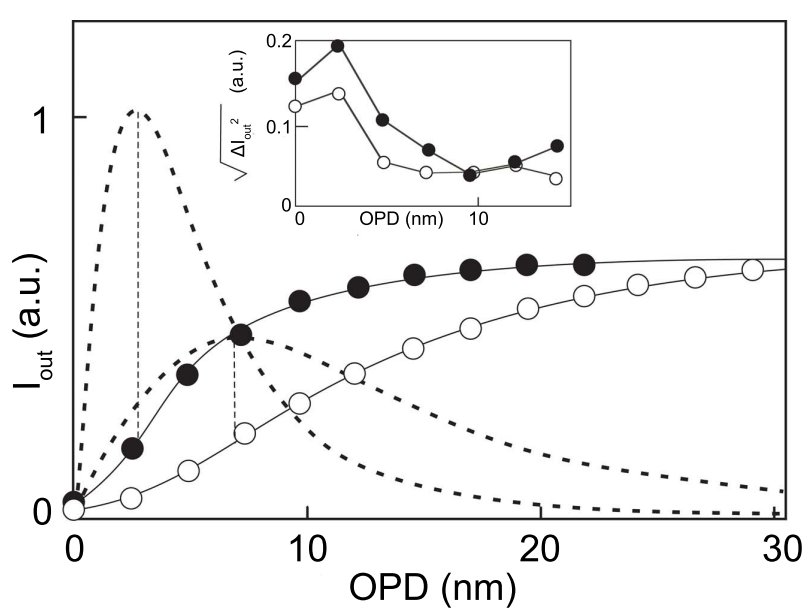

FIG. 3 Measured output intensity $I_{\text {out }}$ as a function of the optical path difference $O P D$, when the cavity is locked to resonance either for the measurement arm (black dots), or for the reference arm (white dots). Solid lines: fits using Eq. (2). Dashed lines: derivatives of the fits. The working points of maximum sensitivity are indicated by the dashed vertical lines. Inset: measured noises associated with the experimental points.

the experimental points are slightly noisier in the more sensitive configuration, the noise ratio being equal to 1.2. For the resolution, one thus gets a net gain equal to $2.3 / 1.2=1.9$. By comparing the sensitivity to the measured noise, we obtain a resolution of $60 \mathrm{pm}$. Finally, we stress that one can always reach the best sensitivity working point by properly adjusting the optical bias, independently of the sample.

The plots of Figure 2 suggest that further increase in sensitivity can be obtained when the interferometer is in the "bright field" configuration, i.e., the two polarizations interfere constructively. Indeed, in that case $\Sigma_{m}$ is three times bigger than in the "dark field" mode, independently of the cavity finesses. However, this configuration is experimentally more challenging because one has to detect small intensity variations over a large DC signal. The measurement therefore needs simultaneously an important detection dynamics and a intensity stabilized laser source. We have experimentally compared the "dark-field" and "bright field" configurations (see Figure 4). We obtain a slope ratio of 2.8 , very close to the theoretical value of 3 . By comparing the noises, reported in the inset of Figure 4, we find that the noise level is 1.5 times higher in bright-field. The overall resolution gain is thus $2.8 / 1.5=1.9$ with respect to the "dark-field" configuration.

\section{CONCLUSION}

The transmission of a resonant two-beam interferometer has been studied in order to measure the optical path variations induced by an intracavity sample. Because of the inherent losses in the sample, the two finesses associated with the cavity eigenstates are different. We have shown theoretically that, when the low finesse eigenstate is servo-locked to resonance, the sensitivity of the apparatus depends only on the high finesse, that is the empty interferometer finesse, thus eliminating the effect of the losses on the measurement. Moreover, a detailed analysis taking into account the different sources of 


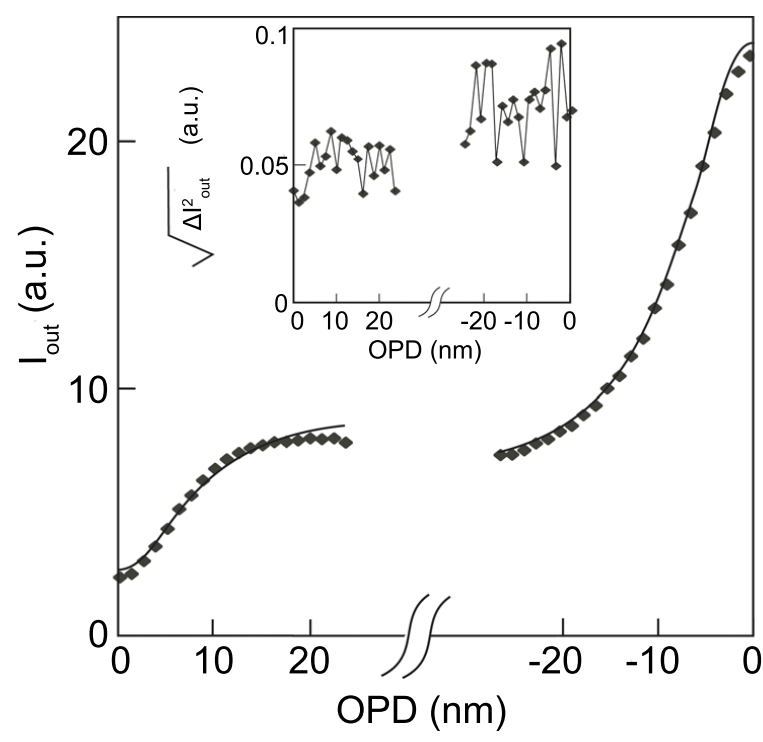

FIG. 4 Measured output intensity $I_{\text {out }}$ as a function of the optical path difference $O P D$ (modulo $\lambda / 2$ ) in the "dark field" and in the "bright field" configurations. Solid lines: fits using Eq. (2). Inset: measured noises associated with the experimental points.

noise in the system demonstrates that using the low finesse eigenstate for cavity locking does not degrade the performance of the interferometer. These properties are confirmed by our experimental results. A minimum detectable optical path difference of $60 \mathrm{pm}$ is obtained for an interferometer with finesse $F=30$, when locking the low finesse peak to resonance with a simple modulation-demodulation servo-loop. We have also shown that this resolution can be further increased by working in the "bright field" configuration. In order to enhance the sensitivity of the apparatus, simple improvements could be performed, such as increasing the cavity finesse and reducing the noise level with a better cavity mechanical stability. To this extent, one may turn to more sophisticated locking schemes which do not require a modulation of the cavity length, such as laser frequency modulation or the FM sidebands technique [12]. Measurements at higher frequency would also reduce considerably the acoustic noise level. Finally, the features we have discussed may find application in the context of other two-beam interferometers, as well as for micro resonators [13] and fiber-based interferometric sensors [14].

\section{ACKNOWLEDGEMENTS}

The authors wish to thank D. Chauvat for preliminary discussions, and A. Carré, L. Frein and C. Hamel for technical assis- tance. This work was performed in the frame of the Contrat de Plan Etat-Région Bretagne.

\section{References}

[1] M. Born and E. Wolf, Principles of Optics (Cambridge University Press, 1999).

[2] N. Arnaud, C. Arnault, M. Barsuglia, M-A. Bizouard, V. Brisson, F. Cavalier R. Chiche, M. Davier, C. Eder, P. Hello, P. Heusse, S. Kreckelbergh, and B. Mansoux, "The global control of the VIRGO experiment" Nucl. Instrum. Meth. A 550, 467-489 (2005).

[3] D. Sigg, "Status of the LIGO detectors" Classical Quant. Grav. 23, $\mathrm{S}_{51}-\mathrm{S}_{5} 6(2006)$.

[4] D. Chauvat, C. Bonnet, A. Durand, M. Vallet, and A. Le Floch, "Jamin Fabry-Perot Interferometer" Opt. Lett. 49, 126-128 (2003).

[5] F. Bretenaker and A. Le Floch, "Laser eigenstates in the framework of a spatially generalized Jones matrix formalism" J. Opt. Soc. Am. B 8, 230-238 (1991).

[6] A. Kastler, "Atomes a l'interieur d'un interferometre Perot-Fabry" Appl. Optics 1, 17-24 (1962).

[7] J. Ye and T. W. Lynn, "Applications of optical cavities in modern atomic, molecular, and optical physics" Advances in Atomic, Molecular and Optical Physics, B. Bederson and H. Walther, Eds., 49, 1 (Academic, 2003).

[8] K. L. Snyder and R. N. Zare, "Cavity ring-down spectroscopy as a detector for liquid chromatography" Anal. Chem. 75, 3086-3091 (2003).

[9] S. T. Logunov, "Cavity ringdown detection of losses in thin films in the telecommunication wavelength window" Appl. Optics 40, 1570-1573 (2001).

[10] G. Ropars, D. Chauvat, A. Le Floch, M. N. O'Sullivan-Hale, and R. W. Boyd, "Dynamics of gravity-induced gradients in soap film thicknesses" Appl. Phys. Lett. 88, 234104 (2006).

[11] M. Vallet, F. Bretenaker, A. Le Floch, R. Le Naour, and M. Oger, "The Malus Fabry-Perot interferometer" Opt. Commun. 68, 423-443 (1999).

[12] R. W. P. Drever, J. L. Hall, F. V. Kowalski, J. Hough, G. M. Ford, A. J. Munley, and H. Ward, "Laser phase and frequency stabilization using an optical resonator" Appl. Phys. B 31, 97-105 (1983).

[13] S. Irmer, J. Daleiden, V. Rangelov, C. Prott, F. Romer, M. Strassner, A. Tarraf, and H. Hillmer, "Ultralow biased widely continuously tunable Fabry-Perot filter" IEEE Photonic. Tech. L. 15, 434-436 (2003).

[14] Z. L. Ran, Y. J. Rao, W. J. Liu, X. Liao, and K. S. Chiang, "Lasermicromachined Fabry-Perot optical fiber tip sensor for highresolution temperature-independent measurement of refractive index" Opt. Express 16, 2252-2263 (2008). 